\title{
The value of the experimental-psychological diagnostics in complex medical care for non- psychotic mental disorders in the elderly
}

\section{Opinion}

The increasing number of elderly patients in the population exacerbates the issues of timely provision them of medical diagnostics and medical-social expertise (MSE) based on accurate diagnosis. ${ }^{1-12}$ According to the survey, sought medical help to psychiatrists, not less than $23 \%$ of them revealed neuro-psychic pathology of vascular origin, mainly non-psychotic mental level. ${ }^{3,4}$ However, the survey did not apply to psychiatrists elderly from the General population indicate that the frequency of shallow ("non-psychotic") of mental disorders of vascular origin in reality is much higher. ${ }^{3,4}$ These patients usually are outpatient by using a common profile medical care, and rarely come to the attention of a psychiatrist. In this regard, significantly increases the relevance of the issues of timely recognition and improve diagnosis and evaluation for the examined disorders in elderly patients. It is important to use an integrated nature of modern MSE, where a psychologist in the composition of the expert committee.

This work is undertaken in order to identify ways to improve the accuracy of diagnostics and MSE in non-psychotic disorders of various origins in the elderly, based on a comprehensive examination, including experimental psychological assessment. At the Department of social psychiatry and psychology, Saint-Petersburg Institute of improvement of doctors-experts examined 36 patients, causing diagnostic and expert difficulties during the examination Bureau of the MSE. The age of patients ranged from 50 to 73years, mean 55.1years. There were 27 men, 9 women.

According to the survey results, 35 of 36 it was recommended that the definition of disability. Social failure, leading to the need for a definition of disability, at patients was associated with non-psychotic neuro-psychiatric disorders of cerebral organic and cerebral vascular Genesis, which was nevrozo- and psychopathic-similar disorder and psychoorganic syndrome. By 1 were discovered post-traumatic Genesis of non-psychotic mental disorders, by 5 - vascular Genesis. At 23 there were mixed (including vascular pathology) cerebral-organic Genesis disorders, another 4 have mixed cerebral organic Genesis of disturbances, but cerebral vascular pathology is not diagnosed at them. In 3 cases diagnosed with other and unspecified origin disorders of cerebral organic nature.

Differences in representation and the role of vascular pathology used to study the significance of different diagnostic methods used in the examined patients. In particular, patients were examined clinically, experimentally psychological, and with the help of psychobiographically techniques. In the survey used data from previously conducted laboratory and instrumental techniques electroencephalography (EEG), etc.

Was investigated the diagnostic significance of clinical, paraclinical (EEG) indices and data of experimental psychological examination (EPE) on the mental processes, to diagnose the presence
Volume 3 Issue I - 2018

\author{
Milutin SM, Sakowskaja VG \\ Professor, Department of Social Psychiatry and Psychology, St \\ Petersburg State IP Pavlov Medical University, Russia
}

Correspondence: Sergey Milyutin M, Professor of the Department of Social Psychiatry and Psychology, St Petersburg State IP Pavlov Medical University, Russia, Email semimi@yandex.ru

Received: December 16, 2017 | Published: January 02, 2018

of non-psychotic mental disorders and their characteristics and Genesis. Statistical correlations between parameters were estimated based on univariate ANOVA, F-test Fisher. Statistical data processing was performed using the computer program SPSS 13.0.

Among the clinical parameters studied data on clinical variants of the psychoorganic syndrome (POS) available in 34 of 36 patients. Asthenic variant POS were present in 4 patients, amnestic variant in 22 , ideational in 11 , explosive in 2 , euphoric in 1 , apathetic in 6. Statistically significant dependence of incidence of amnestic and ideational variants POS from differences in the etiology: the values of the Fisher criterion, respectively, of 32.4 and 4.6.

From paraclinical data indices the results of the EEG. Statistically significant was the distribution of the different EEG depending on varying etiology. Correlation of the distribution of diffuse disorders of the EEG is reflected in the value Fisher criterion $\mathrm{F}=5,7$; correlation of focal distribution of violations is reflected in the value of $\mathrm{F}=4,7$. For violations of EEG stem, diencephalic and cortical localization indices $\mathrm{F}$, respectively, was equal to 2,$8 ; 3,4 ; 5,7$ reflecting sufficient statistical significance of the correlations.

Investigated as indicators of EPE in mental processes - attention, memory, thinking. With the differences in etiology were statistically significantly correlated to the occurrence of persistent deviations from the norm of attention: $\mathrm{F}=4,6$. The occurrence of reversible exhaustion of attention did not depend on the etiological differences: $\mathrm{F}=1,8$. Statistically significantly depended on etiological differences in the occurrence of memory change in the asthenic type, her exhaustion: $\mathrm{F}=2$,9. Statistically not dependent on the etiology of the occurrence of indicators such as changes in memory at the organic type $(\mathrm{F}=1,3)$, exhaustion of thinking $(\mathrm{F}=0,55)$, the evolution of thinking on organic type $(\mathrm{F}=1.2)$. However, not only the presence of cerebral organic pathology was detected at the experimental psychological data on the mental processes-reflected in their weakening, and reduction. Experimental psychological characteristics indicators of this decline has also helped to clarify the etiology, the cause of the lesion, which was confirmed statistically. 
Thus, the present study identified the possibility of further increase of efficiency of diagnostics and medical-social expertise in nonpsychotic mental disorders cerebral vascular and organic origin in the elderly. The obtained data testify in favor of further optimization of medical care to such patients by strengthening its comprehensive character, by using an experimental psychological assessment of cognitive mental processes in addition to conventional clinical and laboratory-instrumental diagnostics.

\section{Acknowledgements}

None.

\section{Conflict of interest}

Authors declare that there are no conflicts of interest.

\section{References}

1. Averbukh ES. Mental disorder at a later age. L Medicine, 1969. p. $1-285$.

2. Voitenko RM, Milutin SM. Methodological principles of medical-social examination and rehabilitation: a teaching manual. 2000. p. 1-48.

3. Gavrilova SI. Modern state and prospects of development of the domestic gerontopsychiatrie Social and clinical psychiatry. 2006;16(3):5-11.
4. Gavrilova SI. Mental disorders in the population of elderly and senile age (clinical-statistical and epidemiological research). Med Sciences M. 1984. p. 1-43.

5. Demyanov YG. Diagnosis of mental disorders: workshop. Moscow Sfera; 2004. p. 1-160.

6. Zharikov NM, Gindikin VY. The prevalence of borderline mental disorders among persons not under the supervision of psychiatric institutions. Journal of neurology and psychiatry. 2002;(7):45.

7. Kalin JB. The mental health of the population of elderly and senile age (clinical-epidemiological study). Med Sciences M. 2001. p. 1-37.

8. Kindras GP. Modern problems of medical-social expertise in mental illness and the ways of their resolution. Med Sciences M. 2001. p. 1-47.

9. Odarchenko SS. Non-psychotic mental disorders of late age. Med Sciences, 2009. p. 1-38.

10. The Federal Law. About social protection of invalids in the Russian Federation of 24 November 1995 N. 181-FZ collected legislation of the Russian Federation. 1995;48:4563.

11. Shakhmatov NF. Mental aging M. 1996. p. 1-304.

12. Sternberg EJ. Geriatric psychiatry. M Medicine. 1977. p. 1-216. 